\title{
ENTRE EL MAR DE BASURA Y LA NUEVA PLANTA DE TRATAMIENTO: MEMORIA HISTÓRICA Y EXPERIENCIAS DIDÁCTICAS POR LA DISYUNTIVA ADMINISTRATIVA DE LOS DESECHOS SÓLIDOS EN RÍO AZUL
}

\author{
Leonardo Bonilla* \\ Stephanie Carballo** \\ Dayanna Madrigal ${ }^{* * *}$ \\ Darwin Membreño ${ }^{* * * *}$
}

Fecha de recepción: 03/02/2016

Fecha de aceptación: 20/04/2016

Resumen: El artículo analiza las implicaciones negativas por el funcionamiento del relleno sanitario y la nueva planta de tratamiento de Río Azul, en la Unión de Cartago, y refiere la experiencia didáctica derivada de la aplicación de un proyecto de acción educativa en la escuela y en dicha comunidad. Por ello, el objetivo se centra en la visualización de las causas de la apertura y el cierre del relleno, así como en los perjuicios socioambientales que aún quedan en la memoria de la población, y que pueden ser tramitados a las nuevas generaciones con fines resilientes; mediante una educación ambiental crítica, que puede considerar las contradicciones en la gestión administrativa de la basura del Estado costarricense, y el daño ocasionado a la sociedad y al medio ambiente. Esta problemática se sigue repitiendo, con algunos peculiares matices, en muchas comunidades costarricenses.

Palabras clave: Educación ambiental, desechos (desperdicios) sólidos, contaminación, vertedero, memoria colectiva, Río Azul, Costa Rica.

\begin{abstract}
This article analyses the negative implications due to the operation of the sanitary landfill and the new treatment plant at Rio Azul, in La Union, Cartago, Costa Rica. The paper also refers the successful teaching experience of implementing an educational action project in the local school and community. Therefore, the objective focuses on making visible the reasons for opening and closing the landfill, as well as on the social and environmental damages still present on the memory of inhabitants, and these prejudices can be inherited to the new generations with resilient objectives. Through a critical environmental education, it is possible to consider the contradictions in the waste management, from the administrative point of view, of the Costa Rica State, and the damage caused to the society and the environment. Issues being repeated with some peculiar nuances in several Costa Rican communities.
\end{abstract}

Keywords: environmental education, solid wastes, pollution, landfill, collective memory, Río Azul, Costa Rica.

\section{Introducción}

\footnotetext{
* Estudiante de la Licenciatura de Enseñanza de Estudios Sociales y Educación Cívica. Escuela de Historia, UNA. Correo electrónico: 1bonilla0805@gmail.com

${ }^{* *}$ Estudiante de la Licenciatura de Enseñanza de Estudios Sociales y Educación Cívica. Escuela de Historia, UNA. Correo electrónico: stefanie_993@hotmail.com

${ }^{* * *}$ Estudiante de la Licenciatura de Enseñanza de Estudios Sociales y Educación Cívica. Escuela de Historia, UNA. Correo electrónico: dayamas02@gmail.com

**** Estudiante de la Licenciatura de Enseñanza de Estudios Sociales y Educación Cívica. Escuela de Historia, UNA. Correo electrónico: darwinni_92@hotmail.com
} 
El Relleno Sanitario de Río Azul, ubicado en La Unión de Cartago, por muchos años se convirtió en un problema ambiental clave en Costa Rica, por el poco progreso estatal en la administración efectiva de los desechos sólidos y líquidos. Pero, ¿cómo nació el mar de basura y cómo tratar las viejas cicatrices de su impacto socioambiental? A propósito de esa y otras preguntas, en las páginas que siguen, se trata de reconstruir la historia del relleno y los nuevos condicionantes sociales y ambientales interpuestos por la nueva planta de tratamiento relocalizada en el lugar, pues se considera, de una forma esencial, la memoria histórica de las comunidades aledañas. Por ello, se propone una acción educativa enfocada en la crítica y concientización de la antropización socioambiental desarrollada por años, y las medidas recilientes que deben ser aplicadas en la nueva población estudiantil, que sufre aún, el perjuicio social por haber vivido cerca del "mar de basura".

Este artículo se constituyó a partir de un trabajo de investigación del curso optativo de Historia Ambiental de Costa Rica, ofrecido a estudiantes de la carrera de Historia, de la Enseñanza de los Estudios Sociales y la Cívica y otras carreras afines que se imparten en la Universidad Nacional (UNA). En este curso se nos insistió en la importancia de complementar investigaciones atinentes a la historia ambiental -reconstruidas mediante estudios científicos en materia ambiental, documentales, revistas, artículos periodísticos y entrevistas $-\mathrm{y}$ concretar acciones educativas.

Conscientes de los avances que el Estado costarricense ha concretado en materia ambiental, también se trata de hacer notar las grandes falencias que todavía se suscitan en las acciones, los programas, los proyectos y las directrices que se dictan para "mejorar" el viejo relleno sanitario y convertirlo en un "venturoso" vertedero, que ha resultado ser el causante de nuevos cambios en el paisaje, y en las lógicas de funcionamiento social y laboral de quienes bucean en él -buzos y buzas es un término popular para referirse a personas que buscan sustento en la basura- $y$ habitantes de sus alrededores, e incluso, en la exposición de conflictos de carácter legal y administrativo por el manejo de los desechos sólidos de la Gran Área Metropolitana (GAM). Situación que se ve reflejada en el descontento de los barrios colindantes al relleno, fruto de las decisiones tomadas por el 
gobierno y las autoridades municipales con las promesas incumplidas de concretar proyectos de desarrollo sustentable.

\section{¿Cómo se desarrolló el problema del mar de la basura en un sitio donde un río azul existió? La historia del Relleno Sanitario de Río Azul}

En Costa Rica, desde la implementación de las medidas económicas y sociales del Estado interventor, a mediados del siglo XX, un modelo desarrollista centrado en originar mejores condiciones de salud, seguridad social, energía, telecomunicaciones, educación, vivienda y agro también prosperó en el ramo de la infraestructura; no obstante, todas la necesidades acumuladas y ulteriores asociadas a los desechos sólidos urbanos quedaron sin ninguna concreción.

Aun cuando en 1961 la población urbana de San José tuvo un impresionante crecimiento demográfico que obligó a las municipalidades de los trece cantones de la GAM a instalar un depósito de basura en la comunidad de Pavas -ubicada a 6,5 kilómetros del Parque Central, en San José-, para sustituir al viejo y agotado basurero de los barrios del sur. Once años después, las quejas de los vecinos de aquella comunidad propiciaron que las autoridades decidieran iniciar una solución conjunta (Avilés Guzmán y Pacheco Urbina, 1995, p. 64), mientras el Estado, tratando de asumir con la mayor seriedad la evidente crisis de desechos sólidos en el Gran Área Metropolitana; gestó un nuevo reglamento para rellenos sanitarios localizados en el país, como un intento por clausurar el relleno agotado.

Como respuesta a las medidas antes tomadas, los trece presidentes ejecutivos de las municipalidades de las provincias de la GAM, firmaron el 25 de abril de 1972, el Convenio Cooperativo Intermunicipal (COCIM), basado en la Ley N. ${ }^{\circ} 4.716$ del Instituto de Fomento y Asesoría Municipal (IFAM), para la “....administración del proyecto de solución conjunta del servicio de recolección, transporte, disposición y tratamiento de desechos sólidos...” (Convenio Cooperativo Intermunicipal, 1993, Art. primero). Dicho acuerdo les permitió decidir el establecimiento de un relleno sanitario en una finca de 62 hectáreas, ubicada en el distrito de Río Azul, una comunidad relativamente despoblada y a una distancia de siete 
kilómetros del centro de San José (Avilés Guzmán y Pacheco Urbina, 1995); aun cuando todavía no se había logrado superar el faltante de camiones recolectores ni se contaba con la maquinaria necesaria para el movimientos de tierra en el futuro relleno sanitario.

Cansada del problema por desechos, el 4 de agosto de 1973, la comunidad de Pavas decidió bloquear el ingreso de los camiones al depósito de basura, que todavía funcionaba en ese lugar, lo que obligó a las autoridades a inaugurar, antes de lo previsto, el Relleno Sanitario Río Azul el trece del mismo mes. Así, la población de San Antonio de Desamparados, distrito por el cual atraviesan los camiones municipales y particulares cargados de desechos sólidos, empezó una nueva dinámica en su vida (Avilés Guzmán y Pacheco Urbina, 1995, p. 66-67).

Toneladas de residuos y el tránsito constante de vehículos empezaron a ser la constante en la zona, lo cual traería consigo la alteración del medio físico y social. Según Avilés Gurzmán y Pacheco Urbina (1995), la circulación de estos medios de transporte se realizaba durante las veinticuatro horas del día, situación que tardó años en poderse regular y establecer un horario reglamentado de seis de la mañana hasta las cuatro de la tarde. A pesar de ello, en 1994 se mantuvo un flujo de circulación de casi ochenta mil vehículos, según se observa en la tabla 1 .

Tabla 1

Ingreso de vehículos al Relleno Sanitario de Río Azul (1994)

\begin{tabular}{ll}
\hline \multicolumn{1}{c}{ Cantón } & Camiones \\
\hline Central & 17.426 \\
Goicoechea & 3.456 \\
Tibás & 2.425 \\
Montes de oca & 2.594 \\
Curridabat & 1.776 \\
Moravia & 1.777 \\
Desamparados & 4.239 \\
Alajuelita & 943 \\
Escazú & 1.402 \\
Coronado & 1.330 \\
La Unión & 1.934 \\
Aserrí & 911 \\
Santa Ana & 817
\end{tabular}

Revista Perspectivas: Estudios Sociales y Educación Cívica - No. 12 
Entre el mar de basura y la nueva planta de tratamiento...

\begin{tabular}{lr} 
Particular & 30.925 \\
Total & $\mathbf{7 1 . 9 5 5}$ \\
\cline { 2 - 2 } & Fuente: Municipalidad de San José. \\
\hline
\end{tabular}

Dicha tendencia se mantuvo en aumento hasta el año 2006, pues como bien se puede palpar, el aumento del volumen de producción de desechos, como resultado del crecimiento de la población, llegó a límites desmesurados -ver tabla 2-.

Tabla 2

Volumen de los desechos recolectados por municipios (1994)

\begin{tabular}{lc}
\multicolumn{1}{c}{ Cantón } & Toneladas métricas \\
\hline Central & 110.042 \\
Goicoechea & 22.070 \\
Tibás & 14.803 \\
Montes de Oca & 12.972 \\
Curridabat & 11.033 \\
Moravia & 10.840 \\
Desamparados & 27.438 \\
Alajuelita & 6.659 \\
Escazú & 8.561 \\
Coronado & 7.214 \\
La Unión & 9.233 \\
Aserrí & 4.045
\end{tabular}


Santa Ana

Total

\subsection{9}

250.039

Fuente: Municipalidad de San José.

El proceso de la entrada en funcionamiento del relleno sanitario inició por la fuerza. El gobierno de José Figueres Ferrer intentó impedir que se desarrollaran manifestaciones de los vecinos y vecinas, quienes se habían organizado desde 1971 a través de su propia Asociación de Desarrollo Integral. Por ello, se abrieron las puertas del relleno y, siete años más tarde, empezaron a darse las primeras invasiones de precaristas al interior de este mismo (Avilés Guzmán y Pacheco Urbina, 1995).

La administración del relleno y su ejecución técnica recayó fundamentalmente sobre la Municipalidad de San José, a pesar de que el COCIM contaba con el reconocimiento y la personería jurídica inscrita en el Registro Nacional. Así, esa municipalidad, mediante algunas modificaciones a las cláusulas del convenio, concretó dichas funciones.

Solo para empezar a narrar los viejos males, los desechos sólidos empezaron a mantenerse destapados por varios meses, y las autoridades municipales del cantón central alegaron que existía una alta tasa de morosidad en el pago de los servicios brindados a las doce restantes municipalidades. Los municipios implicados, a su vez, trasladaron la culpa a los usuarios que tampoco pagan los impuestos destinados a ese rubro (Avilés Guzmán y Pacheco Urbina, 1995).

Unos años más tarde, en 1985, las amenazas de cierre de vías de acceso por parte de los vecinos de Río Azul por la emanación de gases pestíferos y otras demandas más, trataron de frenar el derrame de lixiviados en las calles de la comunidad; situación que conllevó a un estudio topográfico del Ministerio de Salud, en 1987. Esta investigación determinó una vida útil del relleno de siete años. Sin embargo, un año más tarde, se ratificó el COCIM, agravando el problema. Por ello, todas las municipalidades, mediante esa organización, en octubre de 1990 solicitaron, al Poder Ejecutivo, declarar un estado de emergencia nacional, por la inmanejable situación y acumulación de los desechos (Avilés Guzmán y Pacheco Urbina, 1995).

En ese momento, una comisión interinstitucional, creada por el Ejecutivo, recomendó, como la mejor solución, la privatización del relleno en conjunto con el 
transporte de los desechos; ello provocó reacciones encontradas en el círculo político y la sociedad civil, pues el relleno sanitario y su administración fue una oportunidad gratamente recibida por una serie de empresas privadas nacionales y extranjeras que promovían la generación eléctrica a través de desechos, o bien, la producción de gas metano, entre otras alternativas.

Empresas como Tratamientos Biológicos S. A. o Southern Recycling System ofrecieron hacerse cargo del asunto (Avilés Guzmán y Pacheco Urbina, 1995). Por ello no es extraño que el 14 de mayo de 1991 -durante la administración Calderón Fournier- se declarara, según el decreto N. ${ }^{\circ} 20.429$, emergencia nacional el problema de los desechos sólidos, al amparo en la Ley General de Salud; y que, posteriormente, se planteara la modificación del anterior decreto, que pasó de la consideración de Emergencia Nacional a Calamidad Pública, contemplado en la Ley Nacional de Emergencia N ${ }^{\circ} 4.374$.

No obstante, el 19 de octubre de 1995, la Sala Constitucional declaró como inconstitucionales ambos decretos ejecutivos, pues se sospechaba que con ello se pretendía darle otro carácter al dinero destinado al manejo de desechos sólidos (Avilés Guzmán y Pacheco Urbina, 1995).

La mejor forma de mantener abierto y funcionando el relleno sanitario fue negociar con las comunidades una serie de prórrogas de apertura, a cambio de un conjunto de “beneficios” u obras sociales. Al asumir el poder José María Figueres Olsen, en mayo de 1994, se propuso implementar un proyecto de cierre técnico dirigido a realizar una serie de obras al interior del relleno, para convertirlo, una vez clausurado, en un parque ecológico, el cual sería administrado por la Dirección de Parques Nacionales (Avilés Guzmán y Pacheco Urbina, 1995).

El proyecto constaba de varias fases, en la primera se pretendía construir taludes o terrazas para un mejor monitoreo del biogás, para así, posteriormente, dar un mejor tratamiento a los lixiviados; situación que terminaría rehabilitando los terrenos para establecer la infraestructura recreativa del parque.

Con todo y las promesas, la propuesta no se llegó a concretar, a pesar de la participación de instituciones como el Ministerio de Obras Públicas y Transportes, el 
Ministerio de Recursos Naturales, Energía y Minas -ahora MINAE-, el Ministerio de Salud y la Comisión Nacional de Emergencia (Avilés Guzmán y Pacheco Urbina, 1995).

\section{Tratando de reglamentar los desechos sólidos: La búsqueda de soluciones al problema}

A pesar de no concretarse el proyecto de cierre técnico en la administración Figueres Olsen, en el gobierno de Rodríguez Echeverría fue publicado el decreto ejecutivo N. ${ }^{\circ} 27.378-S$, el 23 de octubre de 1998. Su función fue regular las actividades de rellenos sanitarios en la disposición final de desechos para provecho tanto de la población como del medio ambiente (Chaves Barboza, 2008). Según el reglamento, los rellenos sanitarios se clasificarían como manuales o mecanizados y dejaron entendidas las siguientes acepciones y tipologías:

- Relleno manual: método de disposición final de desechos ordinarios de poblaciones urbanas y rurales que generen menos de veinte toneladas diarias de estos desechos; con una vida útil superior a los cinco años, disposición de desechos en capas de veinte a treinta centímetros de espesor para compactación y equipo mínimo para el movimiento y compactación manual de los desechos.

- Relleno mecanizado: método de disposición final de desechos ordinarios de poblaciones urbanas que generen más de cuarenta toneladas diarias de estos desechos; con una vida útil superior a los diez años, sistemas de drenaje de lixiviados y gases, áreas administrativas, taludes finales no mayores a treinta por ciento, disposición de desechos en capas de sesenta centímetros de espesor para compactación, vigilancia y control durante los quince años posteriores al cierre, entre otras especificaciones.

Chaves Barboza (2008) sugirió que, en el caso de poblaciones urbanas y rurales que generen entre veinte y cuarenta toneladas diarias de desechos, podrían utilizarse cualquiera de los dos tipos de relleno. Sin embargo, el reglamento también regulaba la dotación de permisos para la ubicación, la construcción y la operación de los nuevos y modernos espacios de tratamiento; aparte de establecer directrices para evitar la mezcla de ciertos grupos de desechos por su potencial generación a ciertas temperaturas, reacción violenta, fuego, explosión y generación de sustancias toxicas en inflamables. 


\section{El cierre técnico del Relleno Río Azul y la sucesiva creación de la planta de tratamiento}

En el año 2000, la Municipalidad de San José cesó el depósito de sus residuos sólidos en el relleno, trayendo como consecuencia una disminución considerada de ingresos económicos al sitio, pero con la ventaja de que prolongó la vida útil del vertedero. Desde este año y hasta el 2006, el relleno sanitario siguió trabajado hasta su cierre técnico, operado y tratado actualmente por la empresa WPP Continental (Salas, 2000). Antes de su ingreso para la labor de clausura, se llevaron a cabo estudios de diagnóstico y evaluación, realizados por la Secretaría Técnica Nacional, donde uno de sus objetivos fue formular un Plan de Gestión Ambiental del Relleno.

Con el fin de revertir la antropización del espacio, se desarrollaron inspecciones -con visitas de campo, levantamiento fotográfico del terreno, identificación de puntos para muestreos de agua subterránea, medición y análisis de caudales de lixiviados vertidos, mapeo de acuíferos, identificación de áreas susceptibles de deslizamientos y evaluación de sistema de tratamientos- para identificar los problemas provocados por el accionar, o en su

defecto, por la inacción de las administraciones anteriores (Salas, 2000). Por ello, se llegó a la conclusión que en Río Azul, existían los siguientes problemas:

- Mal manejo de lixiviados, lo que acarreó contaminación de las aguas superficiales y subterráneas, pues eran transportados por las cunetas y tragantes pluviales, para finalmente ser vertidos sin control ni tratamiento a las quebradas sur -Río Azul- y oeste -Churuca-.

- Lixiviados evapotranspirados o filtrados en las aguas subterráneas de la zona, equivalente a contaminación provocada por una población de entre 15.500 y 25.000 personas.

- Incorrecto manejo de gases, por el inconveniente sistema de chimeneas para el uso de estañones para tal fin, lo que provocaba el atascamiento, incendio y deterioro de sus conductos.

- Ineficiente manejo de aguas pluviales, por la carencia de acciones prácticas para el control de la erosión en laderas y cunetas de tierra, pues el recubrimiento no existía, por lo que las aguas se infiltraban hacia las zonas profundas del relleno. Incluso, las cunetas 
de concreto en zonas antiguas estaban sin mantenimiento por la pérdida de capacidad hidráulica. Por ello, era muy común enfrentarse a avalanchas de agua, lodo y basura.

- Cobertura de desechos y mecánica de suelos, pues implicó la erosión, la pérdida de la cubierta vegetal y el incremento del arrastre de sedimentos, hundimiento en áreas clausuradas e inestabilidad de taludes.

- Descontrol de la fauna nociva, por la falta de formulación o implementación de programas para el control de la reproducción de perros callejeros y zopilotes, y su permanencia durante todo el día.

- Ausente seguridad personal, industrial y salud ocupacional, porque no existía control en el movimiento de personas, buzos, antes de junio del 2000, así como la desatención de niños y jóvenes dentro del relleno; ni programas para reducir la venta y consumo de drogas, y los asaltos que se habían hecho frecuentes en el predio del relleno.

- Vertido de desechos peligrosos y excesivo movimiento vehicular, por la inexistente autorización controlada del Ministerio de Salud y la Secretaría Técnica Nacional (SETENA), como el protocolo para el manejo, el recibo, la manipulación y la planificación adecuada para los sitios para la disposición de desechos peligrosos de industrias y hospitales; así como la falta directrices orientadas al control y vigilancia de peso en camiones que transitan por las comunidades aledañas o en el propio relleno.

- Participación comunal y problemas sociales, por poca injerencia de las comunidades en la gestión administrativa del relleno y de los proyectos que se propugnaron.

La Federación Municipal Regional del Este (FEDEMUR) -heredera del COCIMcontinuó con los procesos judiciales contra algunos de los ayuntamientos morosos, por concepto de servicio de disposición y tratamiento de los desechos de sus cantones en el relleno sanitario Río Azul (Herrera Castro, 15 de enero de 2008). Incluso el 20 de setiembre del 2001, se publicó en La Gaceta el acuerdo de licitación pública hacia el único oferente, la empresa WPP, cuyo ofrecimiento más tácito fue el cierre técnico en el relleno sanitario de Río Azul, como el propósito de evitar deslizamientos, inundaciones, propagación de vectores, malos olores, daños por la sustracción de bienes públicos, o presencia temporal y permanente de personas no autorizadas (WPP Continental de Costa Rica, s. f.). 
Entre el mar de basura y la nueva planta de tratamiento...

A pesar de las buenas intensiones, transcurrido un año, la Contraloría General de la República revalidó el contrato entre FEDEMUR y WPP por el lapso de tres años, cuya fecha de vencimiento fue el 20 de setiembre del 2005, la cual se negoció desde los estatutos de un nuevo "Convenio de Cooperación para el Cierre Técnico y de Operación del Relleno Sanitario Río Azul”.

Para esa ocasión, la mancuerda entre el Ministerio de Salud y FEDEMUR posibilitó la contratación directa de los servicios de operación diaria de la empresa WPP desde el 17 de agosto del 2006 (Mora, 2008). Por tanto, dicha empresa ha estado laborando en el sitio, con una inversión total que asciende a más de 2000 millones de colones y el siguiente cronograma de trabajo (WPP Continental de Costa Rica, 2002, p. 3) -ver tabla 3-:

Tabla 3

Calendario de trabajo en Río Azul de la empresa WPP (2000-2021)

\begin{tabular}{|l|l|}
\hline \multicolumn{1}{|c|}{ Obras } & \multicolumn{1}{|c|}{ Período de ejecución } \\
\hline $\begin{array}{l}\text { Ejecución de operación mejorada } \\
\text { de disposición en el sitio actual }\end{array}$ & $\begin{array}{l}\text { Desde el 2000 hasta noviembre del } \\
2002 .\end{array}$ \\
\hline Construcción de la ampliación & Marzo 2002 a octubre 2002. \\
\hline $\begin{array}{l}\text { Operación ordinaria del nuevo } \\
\text { relleno y actividades de cierre del } \\
\text { antiguo relleno }\end{array}$ & Noviembre 2002 a diciembre 2005. \\
\hline $\begin{array}{l}\text { Obras de cierre técnico del nuevo } \\
\text { relleno y obras de recuperación } \\
\text { del paisaje }\end{array}$ & Noviembre 2005 a enero 2006 \\
\hline Mantenimiento de post cierre & Febrero 2006 a Febrero 2021 \\
\hline
\end{tabular}

Fuente: WPP Continental de Costa Rica (2002, p. 3). 
Tomando en cuenta la situación actual que enfrenta la planta de tratamiento y sus instalaciones, así como las comunidades aledañas afectadas, los diferentes problemáticas dentro del aspecto ambiental y sanitario continúan vigentes -en menor grado- a pesar de lo planteado y esperado por la empresa encargada del cierre. Por otro lado, analizando la fecha de ejecución propuesta por WPP a la FEDEMUR, otras instancias y público en general, a modo personal se deduce que su cierre integral coincide con la declaración de Costa Rica como país carbono neutral. De esta manera, se cumpliría con la expectativa de los sectores elitistas representados en la clase gobernante al presentar el cierre técnico de Río Azul como un aporte -invisibilizando los conflictos generados desde su apertura- bien recibido por el programa Carbono Neutralidad y la celebración del bicentenario de la independencia.

\section{Educación ambiental para Río Azul: Una propuesta trascendida desde la educación crítica y la acción educativa}

Etimológicamente, la acción de educar, encierra un sinnúmero de labores para la trasmisión de conocimientos en diferentes planos culturales. Sin embargo, cuando se habla de establecer procedimientos concretos para desarrollar una educación ambiental que trascienda la educación formal, las metodologías pedagógicas se complejizan. Máxime si se busca establecer una educación que promueva la participación crítica, coadyuvada con acciones concretas para mejorar el medio ambiente, e incluso, elaborar una cultura ambiental acorde con sus medios de subsistencia; cuyo fin último sea el empoderamiento comunitario para que las poblaciones tomen una importante cuota de poder en el proceso de construcción y mejoramiento ambiental. Mientras en el plano formal, el estudiantado se convierta en sujetos dignos pensadores críticos, capacitados por docentes en un entorno comunal consciente de sus problemáticas ambientales, tejidas a lo largo del tiempo, todo ello condensando en una participación transformadora, justa y sostenible, mediante procesos de aprendizaje recíprocos. 
Entre el mar de basura y la nueva planta de tratamiento...

Pero cómo idear una metodología pedagógica precisa para encarar los procesos socioambientales perniciosos sufridos por años y los nuevos desarrollados por la nueva empresa WPP terminal en Río Azul. La clave sin duda se encontró en los postulados epistemológicos de la teoría crítica y la acción educativa, puesto que la primera se ha constituido en una acepción socio-pedagógica imprescindible para comprender la realidad vivida por las comunidades con problemáticas complejas, como el caso concreto de Río Azul, enfocando en desarrollar el diálogo -para interpretar críticamente el mundo, mediante argumentos y actitudes racionales, tendientes a alcanzar una acción liberadora- y un proceso de concientización social para producir un proceso educativo de transformación social (Rodríguez, Marín, Moreno y Rubano, mayo 2007). Por ello, con el fin de concretar una acción educativa concreta que trascendiera la educación formal -primaria, secundaria y educación superior-, se buscó incursionar en una educación abierta, en busca de una concientización ambiental para la población que habita este entorno, para crear un espacio de discusión de las causas y los efectos perniciosos desencadenados, a través de los años transcurridos con el problema de la basura en la comunidad de Río Azul; procurando crear estrategias y mecanismos para transformar su entorno y mitigar la antropización del espacio, para una mejor planificación de propuestas, metodología y metas a lograr en la educación acción en Río Azul -ver tabla 4-.

\section{Tabla 4}

\section{Planteamiento de acción educativa para Río Azul}

\begin{tabular}{|c|c|c|}
\hline Propuesta & Metodología & Metas a lograr \\
\hline $\begin{array}{l}\text { El Relleno Río Azul } \\
\text { se localiza al suroeste de la } \\
\text { cuidad de San José, en el } \\
\text { distrito de Río Azul, cantón } \\
\text { La Unión. Para la propuesta } \\
\text { de la acción educativa, se } \\
\text { realizó una reseña histórica } \\
\text { que abarca la creación del } \\
\text { relleno hasta la panorámica } \\
\text { actual de dicho lugar con la } \\
\text { planta de tratamientos de } \\
\text { desechos. } \\
\text { Se indagó en las }\end{array}$ & $\begin{array}{l}\text { 1) Elaborar una reseña } \\
\text { histórica de la creación del } \\
\text { relleno sanitario de Río } \\
\text { Azul mediante consulta de } \\
\text { documentos y diarios } \\
\text { nacionales con pertinencia } \\
\text { y énfasis en el problema } \\
\text { de la investigación." } \\
\text { 2) Realizar entrevistas a } \\
\text { las personas lugareñas } \\
\text { para indagar en la } \\
\text { percepción que tienen } \\
\text { sobre el impacto ambiental }\end{array}$ & $\begin{array}{l}\text { - Desarrollar la propuesta de } \\
\text { investigación con } \\
\text { responsabilidad social y } \\
\text { sentido ambiental. } \\
\text { - Crear estrategias para } \\
\text { mitigar el impacto ambiental } \\
\text { y educar al estudiantado en } \\
\text { torno a esta problemática y } \\
\text { concientizar, a nivel local, a } \\
\text { la comunidad del cuido y la } \\
\text { importancia de tener planes } \\
\text { alternativos para el } \\
\text { tratamiento de la basura. }\end{array}$ \\
\hline
\end{tabular}




\begin{tabular}{|c|c|c|}
\hline $\begin{array}{l}\text { formas de crear consciencia } \\
\text { del problema de } \\
\text { investigación y se buscó la } \\
\text { reflexión y la } \\
\text { responsabilidad social y } \\
\text { ambiental con el tema de la } \\
\text { basura en la localidad, para } \\
\text { hacer eco a nivel regional y } \\
\text { nacional. Este análisis y la } \\
\text { reflexión de la } \\
\text { responsabilidad social y } \\
\text { ambiental, iniciaron en el } \\
\text { trabajo de aula con docentes } \\
\text { y estudiantes, por lo cual, } \\
\text { fue de suma importancia la } \\
\text { intervención pedagógica. }\end{array}$ & $\begin{array}{l}\text { en dicho lugar y las } \\
\text { posibles soluciones para } \\
\text { mitigar la problemática. } \\
\text { 3) Consultar y analizar la } \\
\text { dinámica de la planta de } \\
\text { tratamiento y su } \\
\text { importancia para reducir el } \\
\text { daño ambiental mediante } \\
\text { giras de campo. } \\
\text { 4) Crear un planeamiento } \\
\text { de acción educativa a } \\
\text { aplicar en la escuela de la } \\
\text { comunidad de Río Azul. } \\
\text { 5) Realizar charlas en la } \\
\text { escuela y la comunidad, } \\
\text { para poder } \\
\text { retroalimentarse de sus } \\
\text { experiencias e informarles } \\
\text { de las medidas para la } \\
\text { mitigación del problema. }\end{array}$ & $\begin{array}{l}\text { - Inculcar al estudiantado y } \\
\text { personas en general el sentido } \\
\text { ético y responsable con las } \\
\text { comunidades y el ambiente, a } \\
\text { través de la preservación de } \\
\text { este mismo. } \\
\text { - Lograr que dicha } \\
\text { investigación, acompañada de } \\
\text { la propuesta de acción } \\
\text { educativa pueda trascender y } \\
\text { ser aplicada en otras } \\
\text { comunidades con las mismas } \\
\text { o similares problemáticas. }\end{array}$ \\
\hline
\end{tabular}

Fuente: Elaboración propia.

Tal como se sintetiza en el planteamiento de la acción educativa antes descrito, una vez que se tuvo conocimiento de la dimensión histórica del problema socioambiental de Río Azul y aledaños, se buscó crear conciencia en la población estudiantil, mediante un planificado trabajo en las aulas, con docentes y estudiantes, e igualmente, también se desarrolló un proceso investigativo y de formación ciudadana entre pobladores, con el objetivo de identificar impactos socioambientales negativos por la construcción del relleno y de las nuevas dinámicas de funcionamiento implantadas por la planta de tratamiento -los cuales al principio ni ellos mismos tenían muy claro- mediante el diálogo y giras de campo.

La implementación de acción educativa se desarrolló en forma conjunta y complementaria entre dicentes y población comunitaria para iniciar un aprendizaje cruzado -esto incluyó a docentes de primaria y secundaria, estudiantes y población local-, con el fin de generar consciencia de sus problemas, percibidos en diferentes niveles. Por eso se realizaron charlas en la escuela Francisco Gamboa Mora, donde se trabajó con un grupo de tercer año, el cual se mostró deseoso de desarrollar procesos de aprendizaje en los temas 
Entre el mar de basura y la nueva planta de tratamiento...

concernientes a las temáticas ambientales. Partiendo de las problemáticas vividas, se buscó generar ideas propias de cómo mitigar el asunto.

Los resultantes planteamientos y sugerencias los llevamos a líderes comunales, quienes también expresaron nuevos males y recomendaciones para mejorar su situación. Para mayor certeza, decidimos hacer entrevistas a informantes claves, con el fin de acceder a sus miedos resultantes de una violencia simbólica sufrida por años, solo por considerarse parte del problema del mar de basura de Río Azul.

Fue impresionante palpar una agradecida comunidad al mostrar nuestro interés en sus problemas y, a la vez, hacer eco de una conciencia local empodera, pues por muchos años se mostraron bastante pasivos y sumisos ante los avatares de las políticas municipales. Sin duda, se pudo hacer más, sin embargo la sola complementarización entre la teoría crítica $\mathrm{y}$, sobre todo, la acción educativa, trató de ejecutar un proceso educativo responsable, crítico y de participación colectiva, donde los diferentes grupos asumieron y brindaron estrategias para transformar positivamente su entorno. En buena forma, este tipo de acepciones pedagógicas se convierten en una maravillosa oportunidad de involucramiento y retroalimentación ciudadana, con miras a gestar un mejor Río Azul.

\section{Conclusiones}

El tiempo empleado para descubrir el proceso histórico y la aplicación de técnicas educativas innovadoras estuvo motivado, desde el principio, por tejer nuevas lógicas de interpretación del relleno sanitario más polemizado de la historia costarricense, y generar un espacio de concientización y una lectura crítica por parte de la población para fortalecer las asociaciones de desarrollo comunal, influir a las futuras generaciones para exigir a las autoridades pertinentes mejoras en los rellenos sanitarios existentes y fomentar el valor del reciclaje y la separación de residuos.

Máxime que en tiempos de la Costa Rica Verde, empresas encargadas del manejo de residuos sólidos todavía reflejan la laxa burocratización y exigencia real hacia una política realmente sustentable con un vaivén de decisiones intermunicipales, propuestas fallidas y constantes confrontaciones de administraciones para la solución inmediata del problema de los desechos sólidos. Tal vez el mar de basura se acabó en el sitio donde un 
día un río azul existió, sin embargo, el río, la memoria histórica y las nuevas propuestas estatales con respecto a los desechos sólidos continúan vagando sin un punto en común.

\section{Referencias}

Chaves Barboza, G. A. (2008). Generación de un índice de emisiones de metano para el Relleno Sanitario de Río Azul, Costa Rica (Tesis de licenciatura, inédita). Universidad Nacional, Heredia, Costa Rica.

Convenio Cooperativo Intermunicipal. (1993). Documentos constitutivos. San José, Costa Rica.

Avilés Guzmán, A. y Pacheco Urbina, R. A. (1995). Consecuencias sociales del funcionamiento y clausura del relleno sanitario en las comunidades de Río Azul, Tirrases y San Antonio (Tesis de licenciatura, inédita). Universidad Nacional, Heredia, Costa Rica.

Herrera Castro, L. M. (15 de enero de 2008). Municipalidades le adeudan a Fedemur aún $\notin 737$ Municipalcr.com. Recuperado de http://www.municipalcr.com/index.php?option=com content\&view $=$ article\&id $=24$ 5:municipalidades-le-adeudan-a-fedemur-a737-millones-15108\&catid=317:a008$\underline{\text { noticias }}$ 
Mora, G. (2008). Consulta sobre traslado de fondos de presupuesto para obras de cierre técnico al pago de deudas originadas en la operación del Relleno de Río Azul. Carta dirigida a Alexis Rodolfo Cervantes Morales, director ejecutivo del FEDEMUR. Contraloría General de la República, Costa Rica.

Rodríguez, L., Marín, C., Moreno, S. y Rubano, M. (mayo 2007). Paulo Freire: Una pedagogía desde América Latina. Ciencia, Docencia y Tecnología, 34, 129-171. Recuperado de http://www.revistacdyt.uner.edu.ar/

Salas, L. A. (2000). Relleno Sanitario Regional Río Azul, Tirrases de Curridabat y Río Azul de La Unión: Plan de Gestión Ambiental y Declaración de Compromisos Ambientales. Operación Ordinaria, Cierre y Post Cierre. Heredia, Costa Rica.

WPP Continental de Costa Rica. (s. f.). Cierre técnico del Relleno Sanitario Río Azul. WPP Continental. Recuperado de http://www.wppcontinental.com/rioazul.html (2002). Declaración de impacto ambiental sobre Proyecto Ampliación Relleno Sanitario Río Azul y cierre técnico de toda el área de operaciones. (Expediente administrativo $\mathrm{N}^{\circ} 080-2002-\mathrm{SETENA}$ ). San José, Costa Rica. 